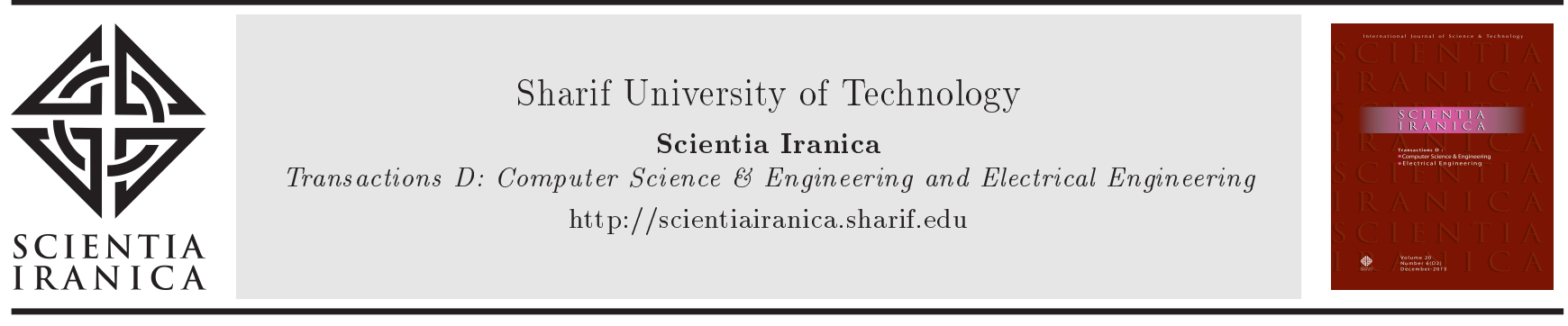

\title{
Modeling of a PIN diode RF switch for reconfigurable antenna application
}

\author{
J. Kumar, B. Basu*, and F.A. Talukdar \\ Electronics and Communication Engineering Department, National Institute of Technology Silchar, Silchar, Cachar-788010, Assam,
} India.

Received 28 February 2017; received in revised form 6 June 2017; accepted 6 January 2018

\author{
KEYWORDS \\ Frequency \\ reconfigurable \\ antenna; \\ PIN diode modeling; \\ Microstrip antenna; \\ SAR investigation; \\ UWB to narrowband \\ switching.
}

\begin{abstract}
The paper presents a procedure to model a PIN diode RF switch using a fullwave EM solver, HFSS. It utilizes the switch to build a simple partial ground plane antenna with an omnidirectional radiation pattern. The proposed antenna has the ability to tune the frequency from the ultra-wideband to narrowband mode. The frequency reconfigurability is accomplished by incorporating a rectangular slot resonator and a PIN diode RF switch embedded in the partial ground plane. The antenna demonstrates $-10 \mathrm{~dB}$ impedance bandwidth from $500 \mathrm{MHz}$ to $6 \mathrm{GHz}$ in the ultra-wideband mode and $1.125 \mathrm{GHz}$ to $2 \mathrm{GHz}$ in the narrowband mode. A parasite is stacked in the ground plane to improve the impedance matching, and a rectangular slot is fused to explore the bandwidth-limiting possibility of the antenna. The optimization of the antenna is done by a full-wave EM solver, HFSS; moreover, Specific Absorption Rate (SAR) of the antenna is examined using the equivalent flat tissue phantom. A prototype of the proposed antenna is fabricated, and execution is accepted by measured results. The proposed antenna has a simple and miniature structure, low SAR $(0.41 \mathrm{~W} / \mathrm{kg})$, an omnidirectional pattern, and low cross polarization $(<-15 \mathrm{~dB}$ in E-plane and $<-30 \mathrm{~dB}$ in H-plane).
\end{abstract}

(C) 2019 Sharif University of Technology. All rights reserved.

\section{Introduction}

The printed monopole antennas have drawn much attention to the variety of communication systems owing to their omnidirectional radiation pattern, UltraWide Bandwidth (UWB), low profile, and ease of fabrication and integration [1]. By using different configurations, numerous printed monopole structures, including rectangular and circular shapes, have been

\footnotetext{
*. Corresponding author. Tel.: +918474801115 E-mail addresses: jayendra854330@gmail.com (J. Kumar); basubanani@yahoo(B.Basu); fatalukdar@gmail.com (F.A. Talukdar)
}

doi: $10.24200 /$ sci.2018.20110 presented in the literature [2-9]. The aptness of the UWB antennas is to replace multiple antennas of a multi-radio system by covering different narrowband services as WLAN, WiMax, GSM, UMTS, Wi-Fi, WMTS, and MICS through a single antenna. However, the design and implementation of UWB systems are complicated due to the significant thermal agitation noise and the requirement of a higher signal-to-noise ratio.

The future communication systems, such as Software Defined Radio (SDR) and Cognitive Radio (CR), are yet under development, and there are no clear standards for them [10]. According to the very First CR standard IEEE 802.22 for Wireless Regional Area Network, sensing and database access are the two prime requirements $[11,12]$. An antenna should 
work in UWB and narrowband modes for spectrum sensing and accessing the data, respectively [13-16]. The current relevance of the antenna design for these developing technologies is to cover different wireless applications. Based on the way of frequency reconfigurability, the electronically frequency-reconfigurable antennas can be broadly classified into two categories: frequency reconfiguration by transfiguring the length of the current path and another by incorporating the slot resonators. In the first case, the RF switches are placed into the radiator of the antenna and are found useful for the narrowband frequency reconfiguration. A multiple-input-multiple-output narrow-multiband frequency reconfigurable antenna was presented in [17], which placed two PIN diode RF switches in the radiator to operate at LTE 20 (791-862 $\mathrm{MHz})$ and LTE 7 (2500-2690 MHz) bands. Similarly, in [18-21], narrowband frequency reconfigurability was achieved by incorporating $\mathrm{RF}$ switches between the current paths. In the second case, electronically controlled slot resonator(s) of rectangular shape, L-shape or of any random shape is incorporated into the radiator or ground plane to achieve the frequency agility. The second mechanism is found useful for wideband and ultra-wideband frequency reconfigurations. In [22], an optimized slot resonator integrated with a varactor diode was incorporated in the radiator to obtain the ultra-wideband to narrowband tunable frequency reconfigurability. In [23], PIN diode integrated slot resonators were embedded in the ground plane to achieve the ultra-wideband to narrowband frequency reconfiguration. However, the second mechanism is also observed for multiband and narrowband frequency reconfigurability $[24,25]$. The work demonstrates the modeling of the PIN diode RF switch in HFSS and its application to frequency reconfiguration from ultrawideband to narrowband using the second mechanism.

In spite of the fact that the reconfigurable antennas are well known, a comprehensive explanation is required to elaborate on the design process using the commercially available EM solver. This paper presents a brief description of modeling a PIN diode RF switch to be utilized for reconfigurable antenna applications. We have studied the performance variations of an ideal switch and a practical switch for a partial ground plane monopole antenna. Further, the antenna structure is optimized through rigorous simulation to achieve wider bandwidth and better impedance matching. In the narrowband mode, the antenna operates in the WMTS band (1.395-1.432 GHz); hence, the SAR of the antenna is examined and noticed under the safety level. The prototype of the antenna is fabricated and simulated, and measured results are found in very good agreement.

Following the introduction, the rest of the paper is organized as follows. The modeling of the PIN diode $\mathrm{RF}$ switch is explained in Section 2. Antenna design and analysis are presented in Section 3. The SAR of the antenna is investigated using the simulation model in Section 4. A prototype of the proposed antenna is fabricated and measured results are discussed in Section 5. Finally, the work is concluded in Section 6 .

\section{Modeling of PIN diode RF switch in HFSS}

Different electronic components, such as PIN diodes, FETs, and MEMS, are used to build an RF switch for frequency reconfigurable antennas [17,26,27]. The MEMS-based RF switches are useful in the cases where low loss and high isolation are required, although they are costlier and require higher operating voltage. However, the FETs have low power consumption, yet suffer from higher loss and poor linearity. The PIN diode-based RF switches are cheaper and offer low-loss operation. The limitation of a PIN diode switch is that a forward dc current is required to switch the diode in the ON state, leading to poor radiation efficiency. In this section, the modeling of a PIN diode RF switch is graphically demonstrated using HFSS. Insertion loss and isolation are the key parameters of RF switches. These parameters depend not only on the devices but also on the configurations of biasing circuits. For diode-based RF switches, series and shunt connected diodes are the most commonly used configurations [28]. However, due to the ease of integration, most of the reconfigurable antennas reported in the literature have used the series configuration of the RF switch and external biasing circuits. Here again, BAP64-02 pin diode-based series configuration of the RF switch is utilized. The configuration of the RF switch [28] and the insertion and isolation loss are shown in Figure 1. The RF switch is simulated by Advanced Design System (ADS).

The equivalent circuits of a PIN diode RF switch in ON and OFF states are shown in Figure 2(a) and (b), respectively [28]. The equivalent circuits of the PIN diode RF switch in ON and OFF states can be modeled in HFSS by assigning "Lumped RLC" boundary to the 2D- or 3D-structures [29]. In the HFSS, a single 2Dor 3D-structure can be modeled as a single lumped element or a parallel combination of lumped elements (resistor, inductor, and the capacitor). Similarly, for a series combination of the $n$-number of elements, $n$-number of interconnected structures are required. In the ON state, the equivalent circuit consists of a series combination of two DC blocking capacitors, an inductor, and a resistor.

Similarly, the OFF-state equivalent circuit has a series combination of four different elements. Thus, four interconnected 2D- or 3D-structures are required to model a PIN diode RF switch, as shown in Figure 2(c). Now, to model a structure as a lumped component, "Lumped RLC" boundary is assigned to 


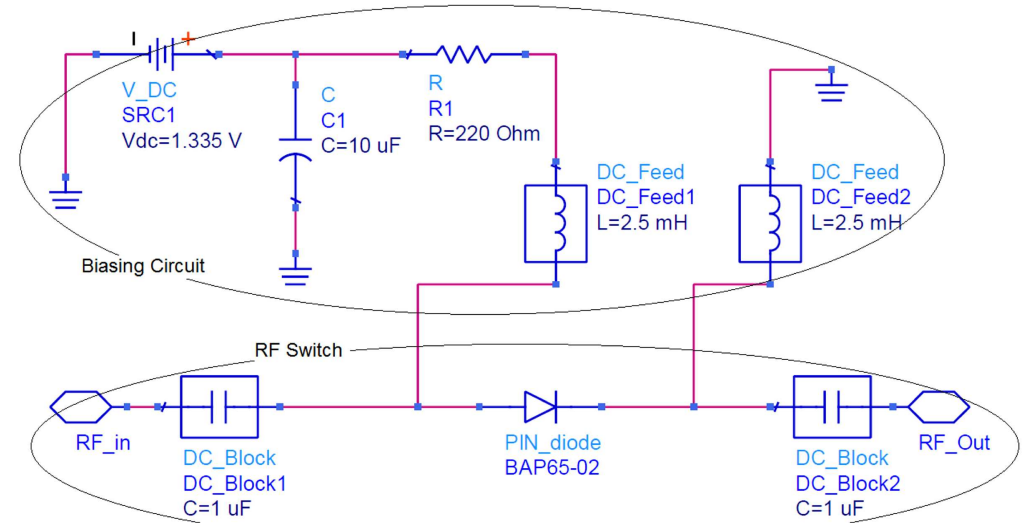

(a)

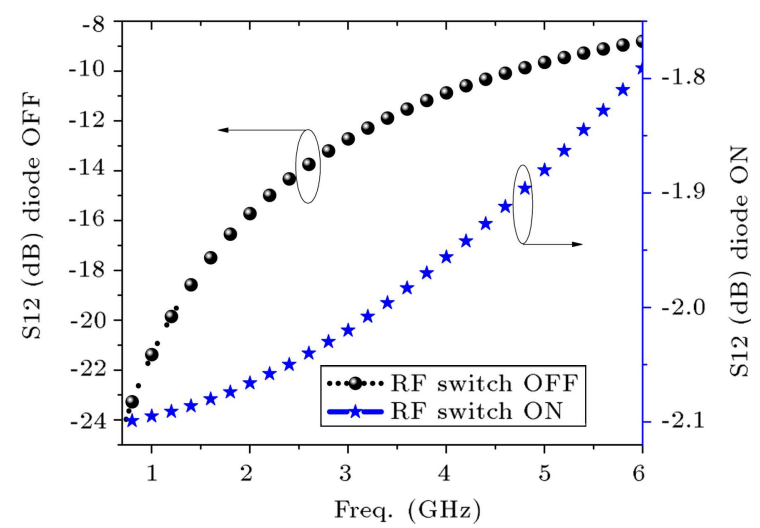

(b)

Figure 1. Configurations of the PIN diode RF switches: (a) Configuration and (b) performance.

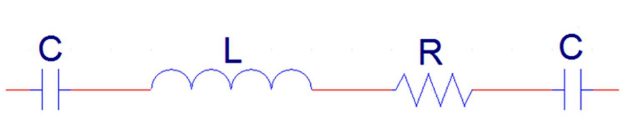

(a)

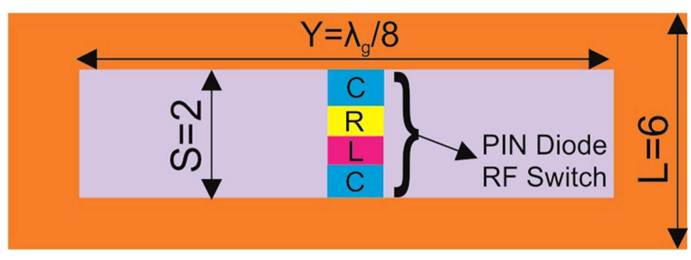

RF switch ON

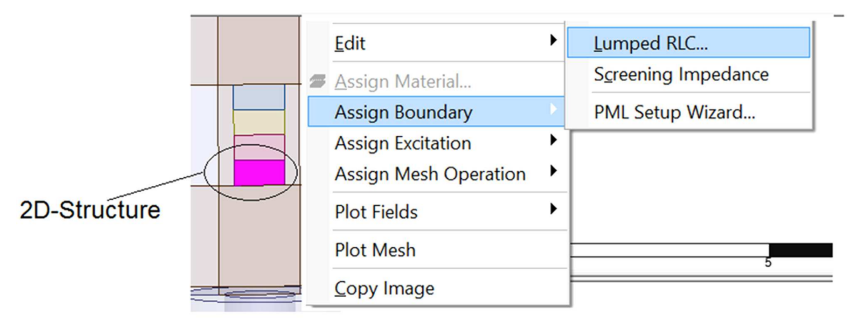

(d)

Figure 2. Modeling of the PIN diode RF switch in HFSS: (a) RF switch ON-state equivalent circuit, (b) RF switch OFF-state equivalent circuit, (c) four interconnected 2D-structures representing switch model in HFSS, and (d) "Lumped RLC" boundary assignment to a 2D-structure (all dimensions in $\mathrm{mm}$ ).

each structure. To assign the boundary, the direction of the current flow should be defined, as shown in Figure 2(d). To model the PIN diode RF switch in the ON state, two blue 2D-structures are assigned as a capacitor (C, blue), pink as an inductor ( $\mathrm{L}$, pink), and yellow as a resistor ( $\mathrm{R}$ or $\mathrm{R} / / \mathrm{C}$, yellow), (c)
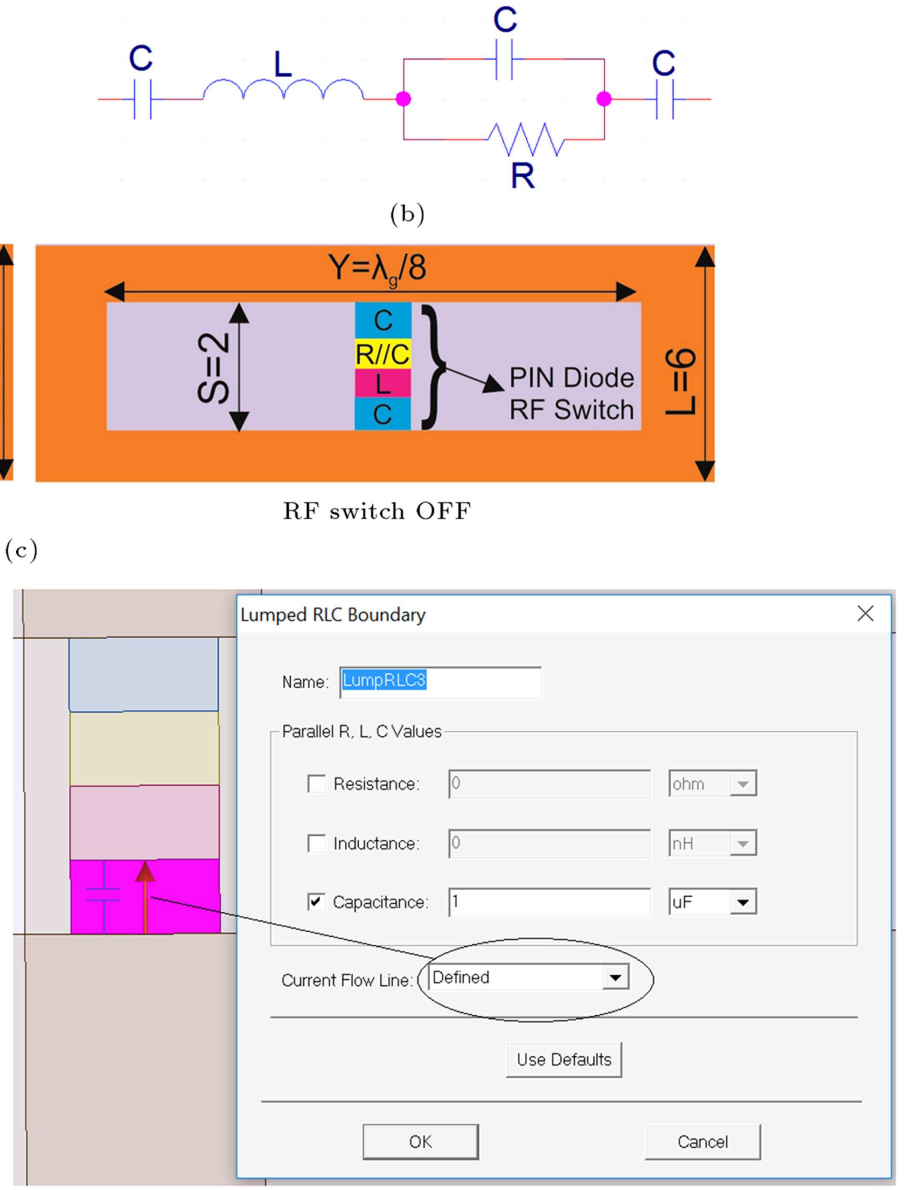

(b)

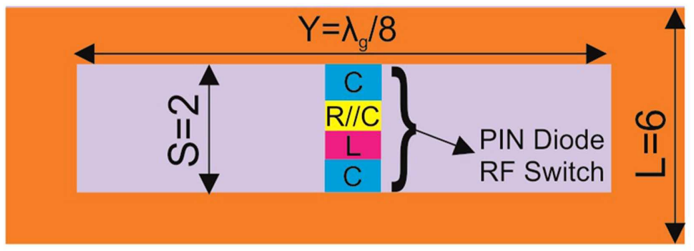

RF switch OFF

as shown in Figure 2(c). In the OFF state, both resistor and capacitor values are assigned to the yellow 2D-structure, and the other structures are kept as they are. Further, the lumped RLC assignment can be modified simply through "boundaries" in Project three. However, the size of $2 \mathrm{D}$-structures is taken by 


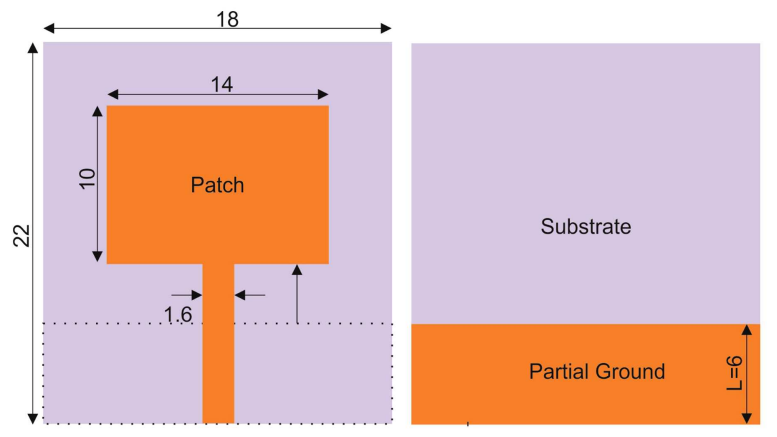

(a)

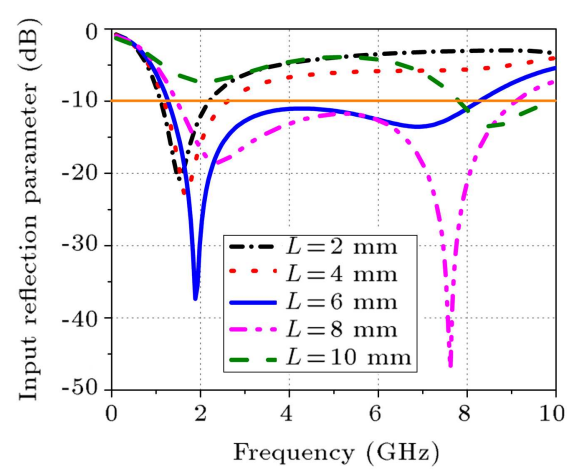

(b)

Figure 3. (a) Basic partial ground plane monopole antenna configuration and (b) input reflection parameter versus length $(L)$ of the partial ground plane (all dimensions are in $\mathrm{mm}$ ).

considering the size of practical components, ease of soldering, and integration of the diode biased lines.

\section{Antenna design and analysis}

A monopole rectangular microstrip antenna is chosen as a basic radiating structure (Figure 3(a)) on account of its ultra-wide bandwidth and good radiation characteristics [2-9]. The antenna is built on FR4 Epoxy dielectric substrate with dielectric constant of 4.4 , dielectric loss tangent of 0.018 , thickness of $1.57 \mathrm{~mm}$. The antenna is excited via $50 \Omega$ inset feed through an SMA connector.

The length of the partial ground $(L)$ is optimized to obtain ultra-wide bandwidth, as shown in Figure 3(b). Due to better impedance matching at the lower cut-off frequency, $L=6 \mathrm{~mm}$ is chosen for the final design. It is clearly observed that the ground plane length $(L=6 \mathrm{~mm})$ shifts the lower edge of the frequency band to its minimum, preserving the length of the current path and presenting the best input scattering parameter (S11) at the lower cut-off frequency (Figure 3). The frequency reconfigurability is realized by integrating a bandstop filter in the partial ground, as shown in Figure 4 . The bandstop filter comprises $\lambda_{g} / 8\left(\lambda_{g}\right.$ is the guided wavelength) rectangular slot resonator and an RF switch embedded in the center

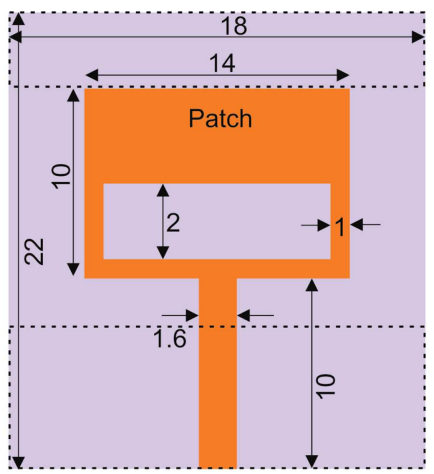

(a)

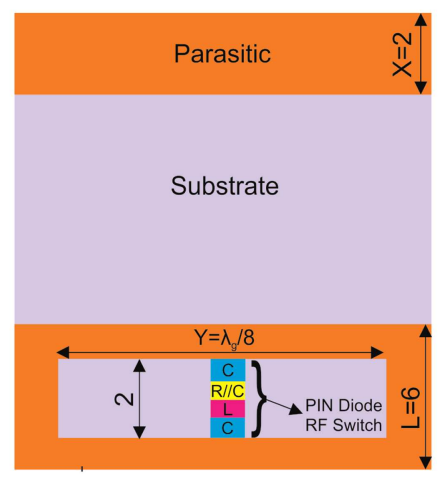

(b)
Figure 4. Configuration of the proposed antenna: (a) Top view and (b) bottom view (all dimensions are in $\mathrm{mm}$ ).

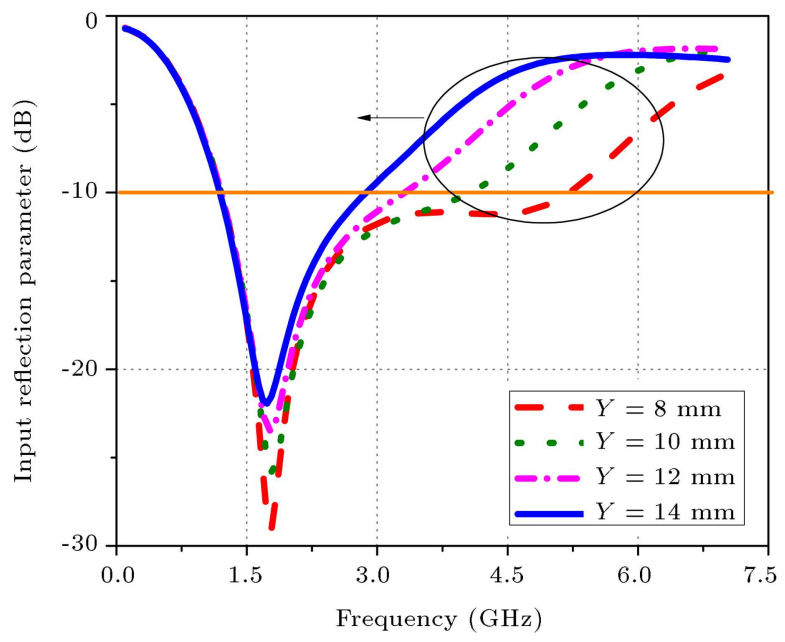

Figure 5. The $\lambda_{g} / 8$ resonator incorporated in the ground plane of the UWB antenna exhibiting the contraction of the bandwidth with increasing slot length.

of the resonator. It is shown in Figure 5 that as the length of the CPW bandstop resonator increases, the bandwidth of the antenna becomes narrower. In this way, by integrating the filter and antenna together, the narrowband operation is obtained.

The antenna operates in the ultra-wideband mode as the filter is disabled by placing the switch in the ON state and yields single band operation as the filter is enabled by turning the switch to the OFF state. The bandstop behavior of the filter can be explained by the surface current distribution, as shown in Figure 6 .

When the switch is in the ON state, it provides a direct path to the current causing the antenna to operate in the ultra-wideband mode. As the switch is turned OFF, the current path through $\lambda_{g} / 8$ resonator becomes dominant, and the antenna operates in the narrowband mode. To obtain better impedance matching, a parasite is loaded on the ground, as shown in Figure 4 . The length $(X)$ of the parasite is optimized to improve the S11 parameter, as shown in Figure 7. However, there is no significant change in the 

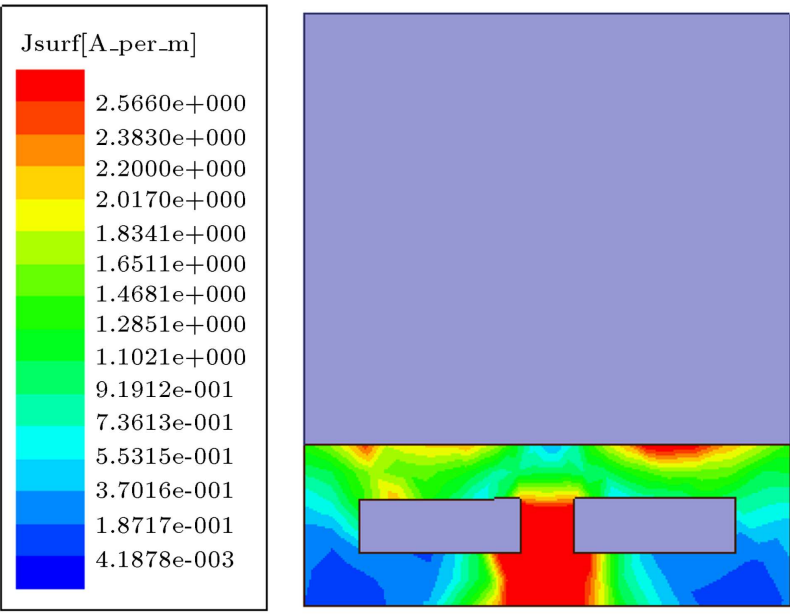

(a)
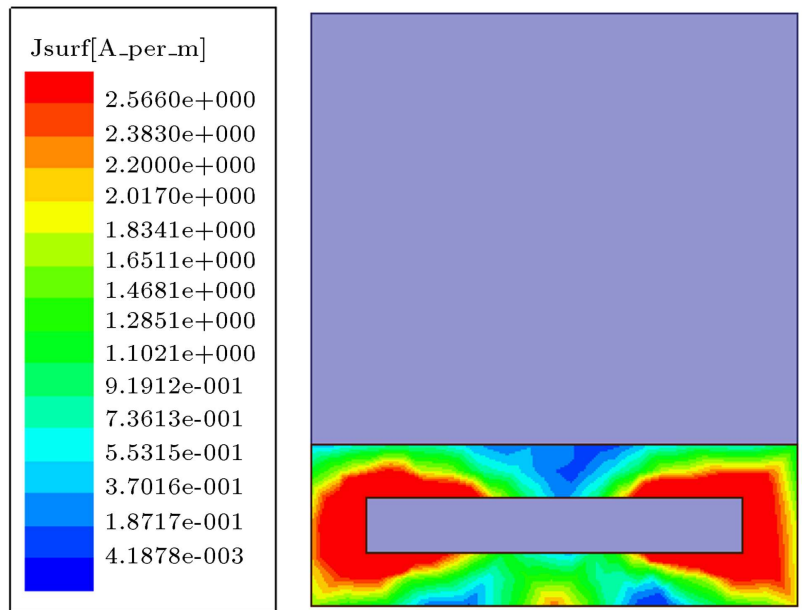

(b)

Figure 6. Surface current distribution on the partial ground plane with an embedded slot resonator at 1.43 GHz: (a) Switch in the ideal ON state and (b) switch in the ideal OFF state.

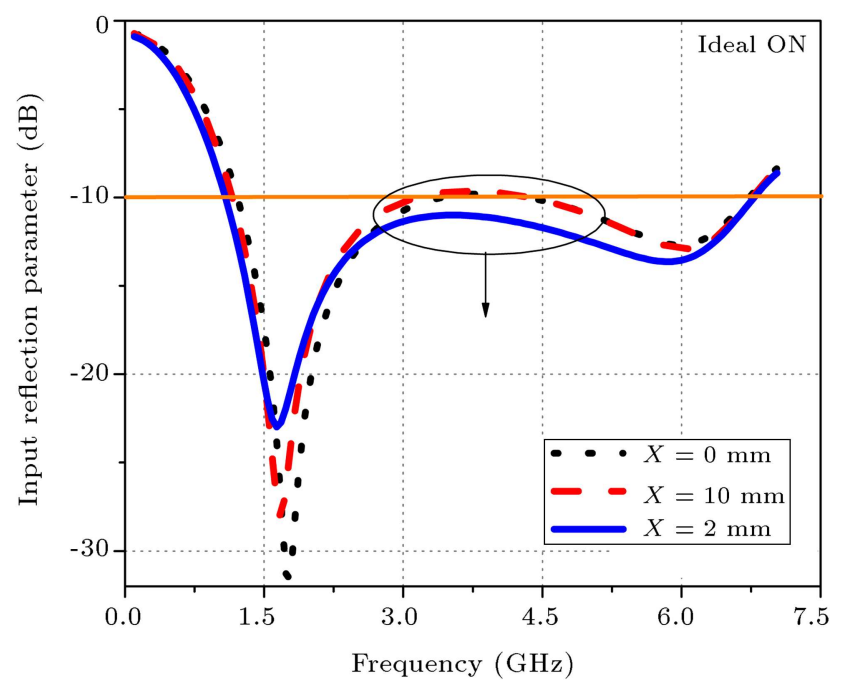

Figure 7. Input reflection parameter of the proposed antenna for different lengths of loaded parasite (all dimensions are in $\mathrm{mm}$ ).

impedance matching characteristics of the narrowband mode.

The antenna operates in the ultra-wideband mode as the filter is disabled by placing the switch in the ON state, and it yields single band operation as the filter is enabled by turning the switch in the OFF state. The bandstop behavior of the filter can be explained by the surface current distribution, as depicted in Figure 6 . When the switch is in the ON state, a direct path is provided to the current, causing the antenna to operate in the ultra-wideband mode.

The $-10 \mathrm{~dB}$ impedance bandwidth of the proposed antenna is investigated for the ideal switch and a practical PIN diode RF switch, as depicted in Figure 8. A perfect electric conductor is used to realize the ideal switch in the ON state and open

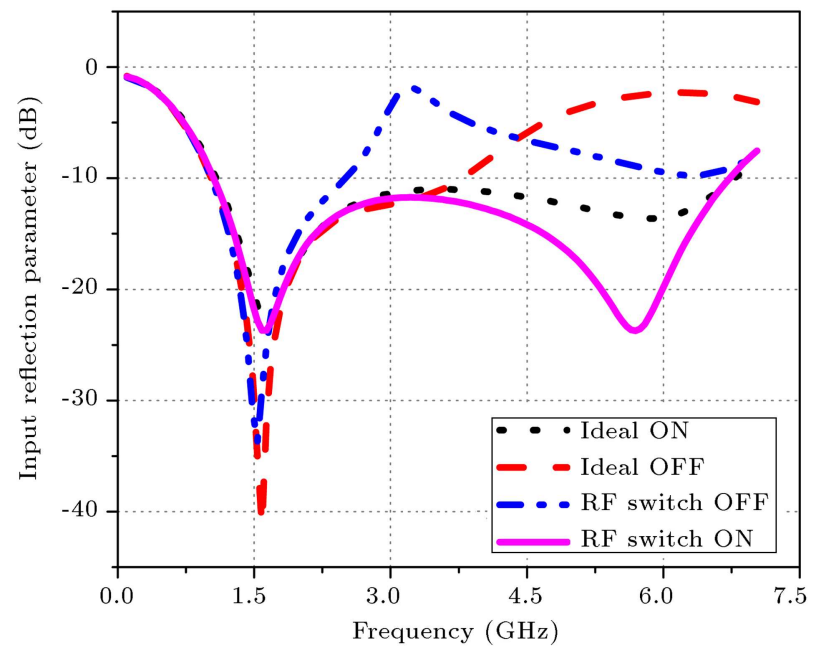

Figure 8. Input reflection parameter of the proposed antenna for the ideal switch and practical switch.

circuit in the OFF state. A practical PIN diode is modeled as a $1 \Omega$ resistor connected in the series with $0.6 \mathrm{nH}$ inductor. In the OFF state, the diode is modeled as a $0.6 \mathrm{nH}$ inductor connected in the series with a parallel combination of $0.5 \mathrm{pF}$ capacitor with a $20 \mathrm{~K} \Omega$ resistor [30]. Two capacitors of $1 \mu \mathrm{F}$ are placed back and forth to the PIN diode to provide the RF connectivity and block the dc. For a practical switch, the antenna exhibits narrower bandwidth in the narrowband mode and better impedance matching in the UWB mode over an ideal switch. The inductance, ON-state resistance, and OFF-state capacitance are taken from the technical data sheet of BAP65-02, 115 PIN diodes.

UWB antennas exhibits a number of advantages: high-rate data support, high security, usefulness for multiple radiosystems, and low power consumption. However, the structure suffers from high on-board- 


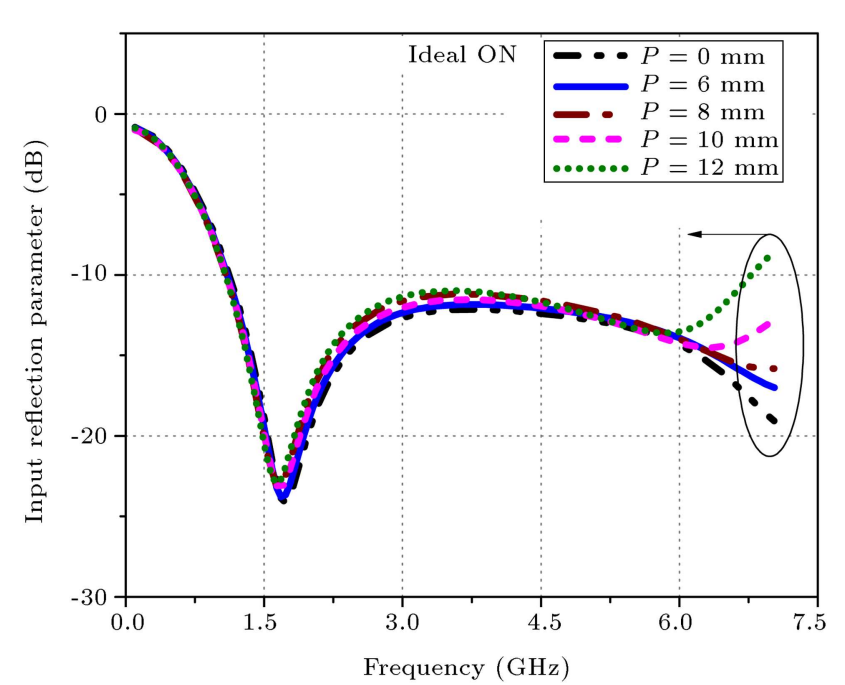

Figure 9. Input reflection parameter of the proposed antenna as a function of the length of the embedded rectangular slot in the radiating patch.

noise, complicated wideband channel characteristics, and matching network and LNA design. Therefore, an additional slot resonator is incorporated in the radiating patch to study the bandwidth-limiting possibilities of the ultra-wideband antenna, inspired by [9] It is observed that loading the slot in the radiating patch also results in the contraction of the bandwidth, although it is seen in Figure 9 that the contraction of the bandwidth is not significant. The inclusion of the rectangular slot does not significantly affect the matching characteristics in the narrowband mode.

\section{SAR evaluation}

The proposed antenna covers WMTS band in the narrowband mode. However, medical telemetry devices, typically used within the $20 \mathrm{~cm}$ range of the body, are required to be investigated for the safety level, which is defined by SAR. In this section, the SAR of the proposed antenna is investigated using a simulation model. Different fragments of the human body are modeled as an equivalent flat phantom of $33 \times 22 \times$ $18 \mathrm{~mm}^{3}$ dimension. The local SAR of the muscle phantom at the separation of $5 \mathrm{~mm}$ is studied, as shown in Figure 10. The small separation between the antenna and the human body significantly changes the

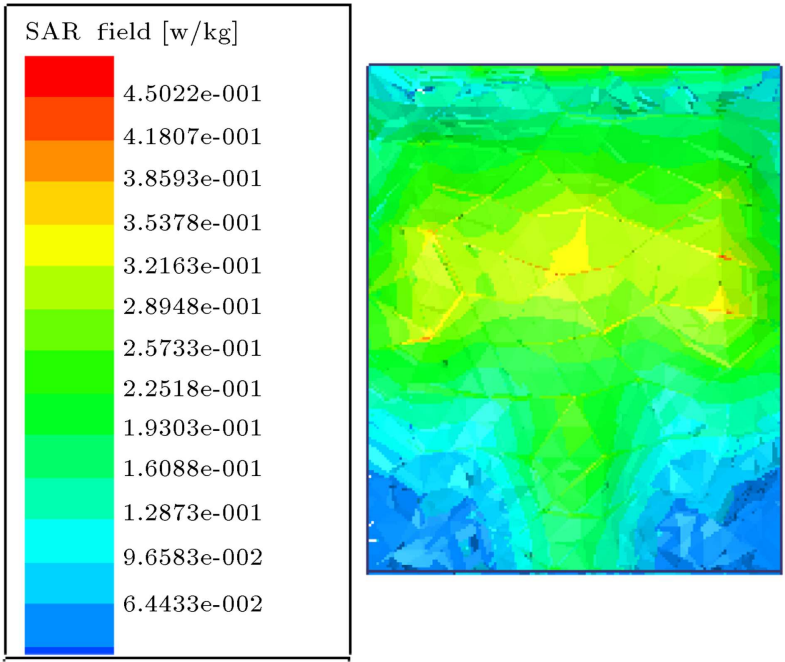

Figure 10. Local SAR on a muscle equivalent flat phantom $\left(22 \times 18 \times 10 \mathrm{~mm}^{3}\right)$ at $1.43 \mathrm{GHz}$.

effective dielectric constant of the antenna, resulting in frequency deviation (Table 1 ). In the investigation of the SAR of the proposed antenna, the 1-g-averaged SAR of the antenna is found under the safety level (IEEE C95.1 1999, SAR $\leq 1.6 \mathrm{~W} / \mathrm{kg}$ ) for $9 \mathrm{~mW}$ input power.

\section{Implementation and measured results}

A prototype of the proposed antenna is fabricated, as shown in Figure 11, and performance parameters are measured. For the sake of simplicity, a simple resistive biasing circuit is used to bias PIN diode NXP BAP65-02, 115. However, the RF chokes can be used to improve the isolation of the switch. Two $0.1 \mu \mathrm{F}$ capacitors are connected in the series to the PIN diode to establish the RF connection and, also, to block the dc.

The intrinsic region of a PIN diode accommodates a high concentration of charge; hence, a very shortduration negative cycle of an RF signal cannot reverse the diode-biasing, which allows the switch to act bilaterally at RF frequencies. By connecting the dc supply, the bandstop filter is disabled to operate the antenna in the UWB mode. As the biasing voltage is disconnected, the filter is enabled, allowing the antenna

Table 1. Simulated results of SAR analysis.

\begin{tabular}{lcccc}
\hline Tissues & $\varepsilon_{\boldsymbol{r}}$ & $\boldsymbol{\sigma}(\mathbf{S} / \mathbf{m})$ & $\mathbf{B W}(\mathbf{G H z})$ & Peak SAR $(\mathbf{W} / \mathbf{k g})$ \\
\hline Air & 1 & 0 & $1.2-2.5$ & - \\
Muscle & 54.066 & 1.1553 & $1.1-1.88$ & 0.45 \\
Skin dry & 39.59 & 1.0465 & $1.08-1.9$ & 0.32 \\
Bone & 12.03 & 0.2156 & $1.09-2.03$ & 0.23 \\
Body phantom & - & - & $1.1-1.9$ & 0.41 \\
\hline
\end{tabular}




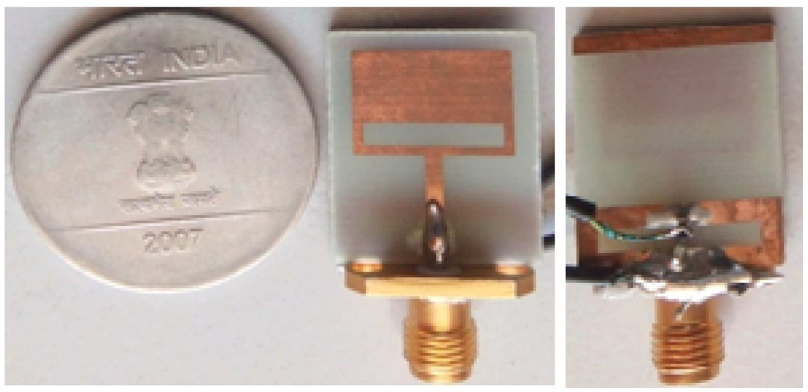

Figure 11. Fabricated prototype of the proposed antenna (from the left: one Rupee Indian coin, top view of the antenna, and bottom view of the antenna with embedded PIN diode RF switch).

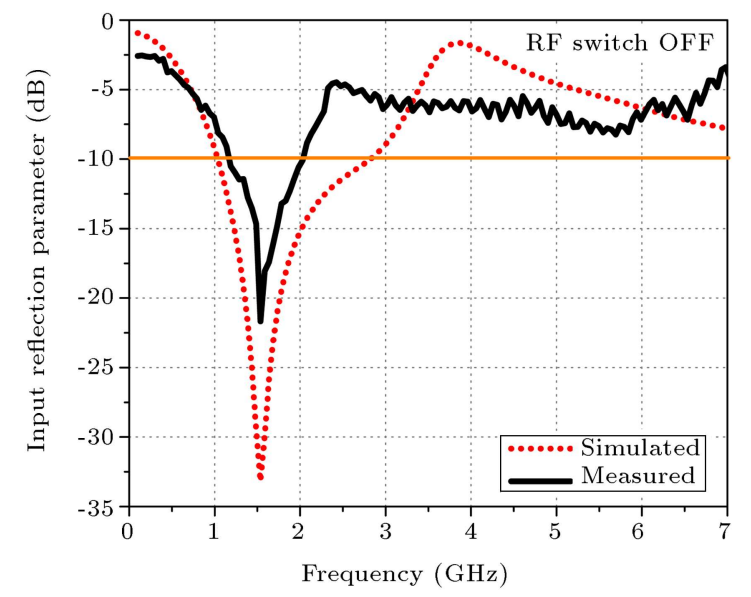

(a)

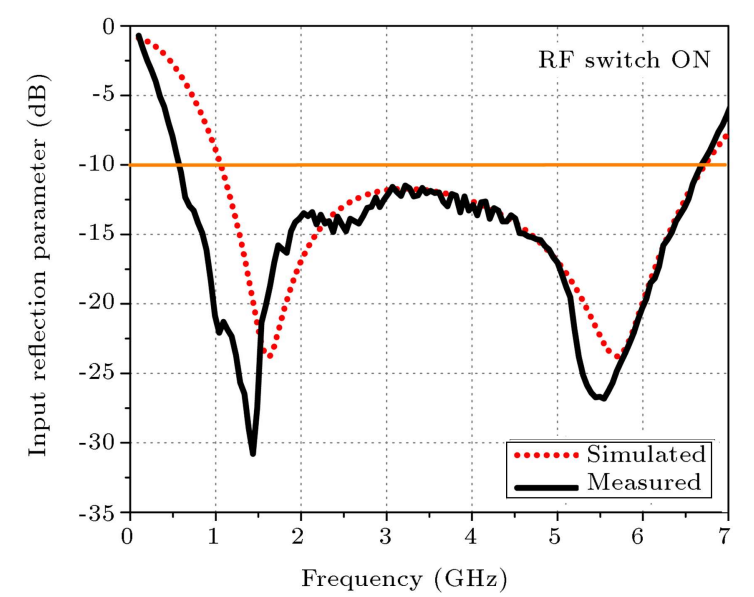

(b)

Figure 12. Simulated input reflection parameters of the proposed antenna: (a) PIN diode RF switch in the OFF state and (b) PIN diode RF switch in the ON state.

to operate in the narrowband mode. The simulated and measured input reflection parameters of the antennas are found to be in very good agreement, as shown in Figure 12. The antenna has an omnidirectional pattern and sufficiently low cross polarization in the Eplane and very low cross polarization in the H-plane, as
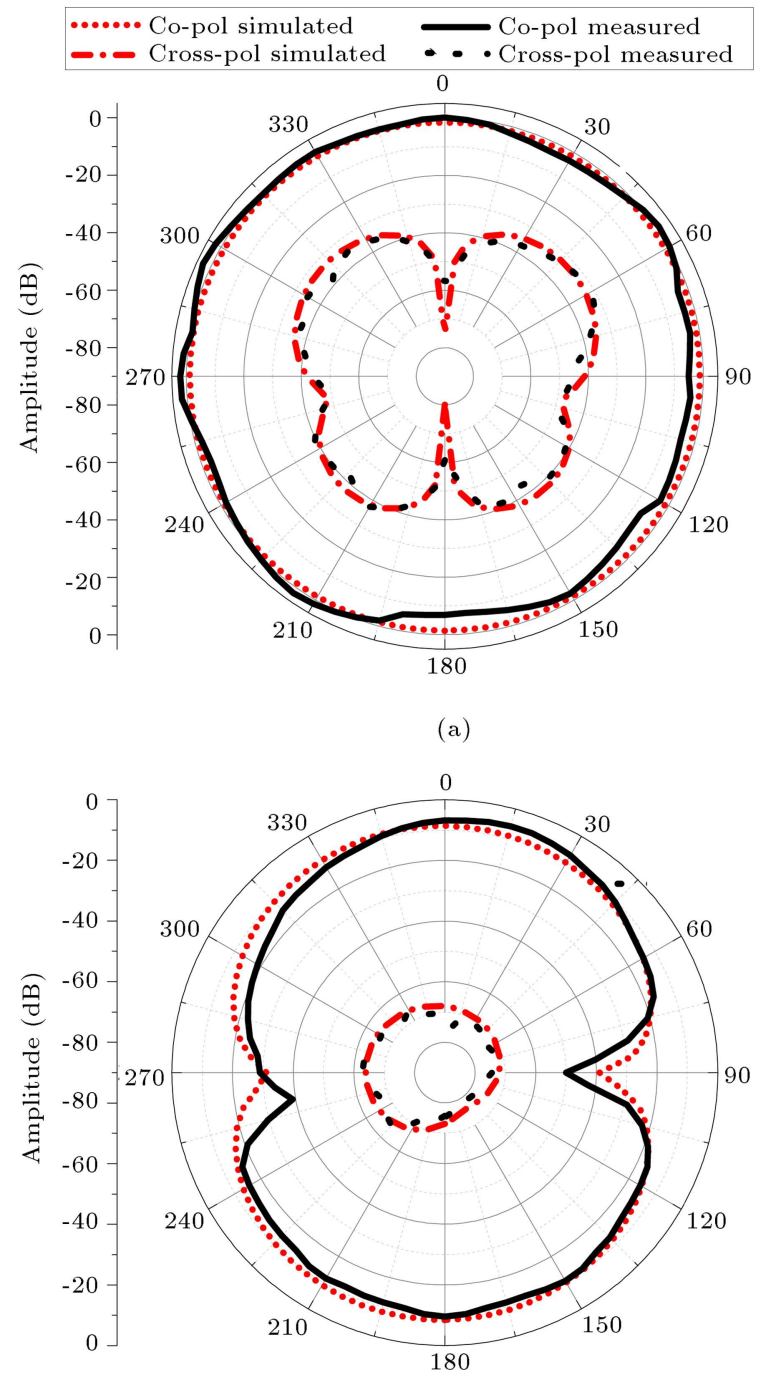

(b)

Figure 13. Radiation pattern of the proposed antenna at $1.43 \mathrm{GHz}$ : (a) Uniform distribution of the radiated power in the azimuth plane (E-plane) and (b) power radiating only for a sector of the elevation plane (H-plane).

depicted in Figure 13. The antenna achieves the peak realized gain of around $2.5 \mathrm{dBi}$ in the UWB mode and $-3 \mathrm{dBi}$ in the narrowband mode, as shown in Figure 14.

However, in the literature, it is shown that the antennas with a gain of $-4 \mathrm{dBi}$ work well for applications like RFIDs [31]. The identical characteristics of the antenna gain in ON and OFF states can be explained by the surface current distributions on the radiator. The surface current distributions on the radiator in the narrowband and UWB modes are identical, as shown in Figure 15, leading to an identical gain characteristic. Moreover, in the OFF state, there is a decaying notch at $3.1 \mathrm{GHz}$ in the gain characteristics. To examine this effect, the surface current distributions on the radiator are studied for the edges of the notch $(2.5 \mathrm{GHz}$ and $3.5 \mathrm{GHz})$, as shown 


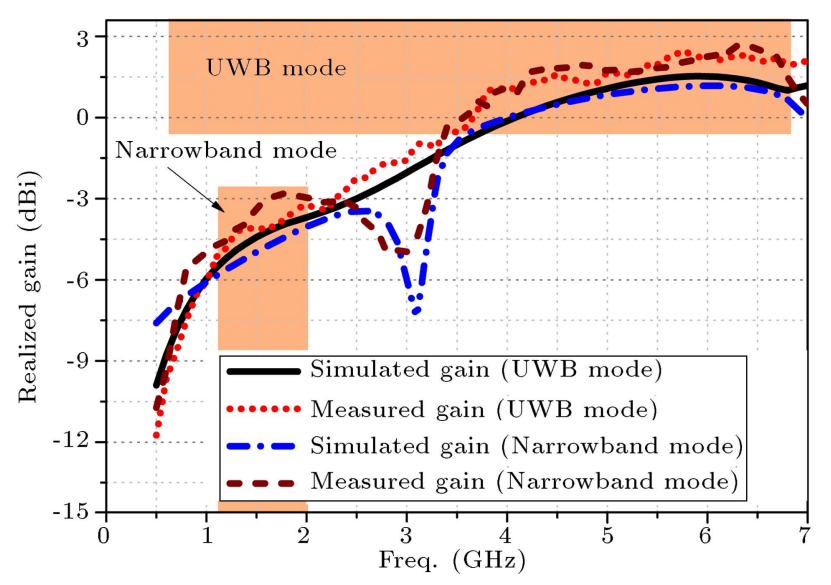

Figure 14. Realized simulated and measured gains of the proposed antenna in the UWB and narrowband modes.

in Figure 16. It is found that, at $3.1 \mathrm{GHz}$, the surface current distribution is extremely poor as compared to the edges resulting in poor radiation, although the main result of using the switch between the modes is the frequency reconfigurable characteristic; further, radiation enhancement techniques can be employed to improve the performance of the proposed antenna.

\section{Conclusion}

The procedure for modeling a PIN diode RF switch using HFSS was demonstrated, and the switch model was utilized to design a simple monopole antenna with two switchable states. The PIN diode RF switch provided sufficient isolation in the OFF state to run the antenna in the narrowband mode. It was observed that incorporating an RF switch significantly degraded the antenna gain for a particular band $(3 \mathrm{GHz})$. However, the radiation performances of the other operational band were unaffected. In the UWB mode, the antenna covers different narrowband services such as WMTS, UMTS, and LTE. In the narrowband mode, only applications operating in L-band (1-2 GHz) as WMTS are covered with very good impedance matching. By virtue of the simple structure, frequency agility, omni-
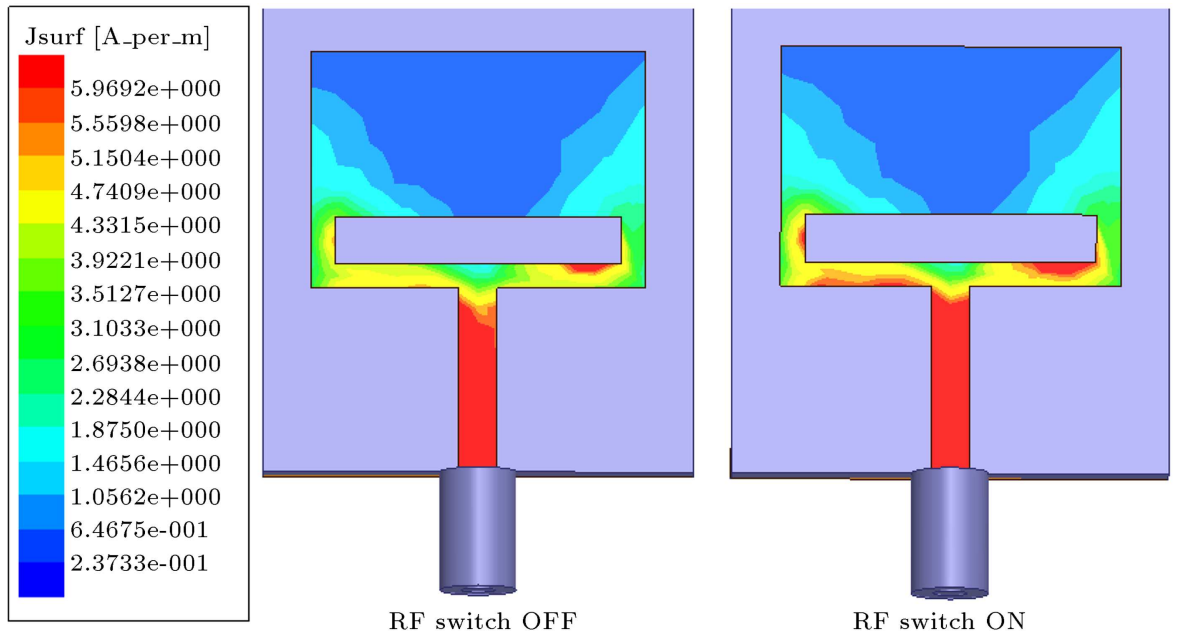

Figure 15. Surface current distribution on the radiator in the ON and OFF states of the switch at $1.43 \mathrm{GHz}$.

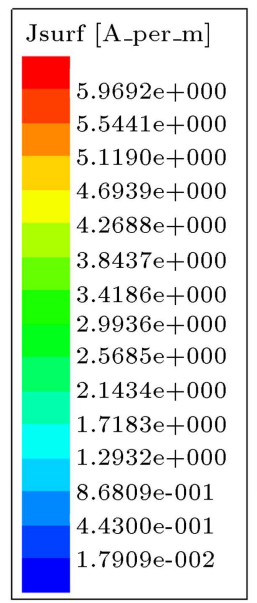

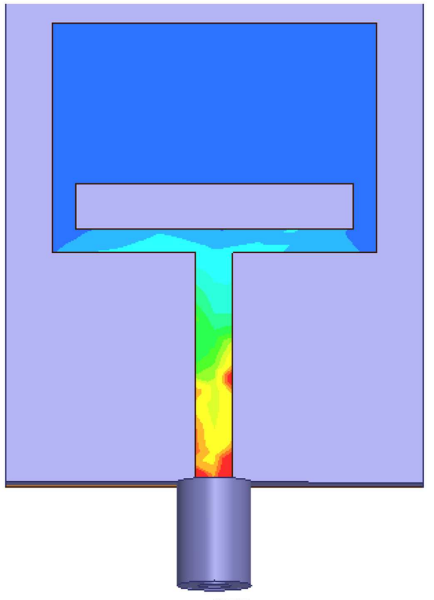

$2.5 \mathrm{GHz}$

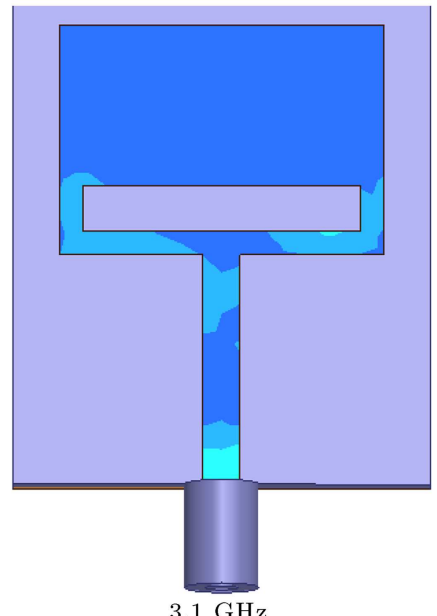

$3.1 \mathrm{GHz}$

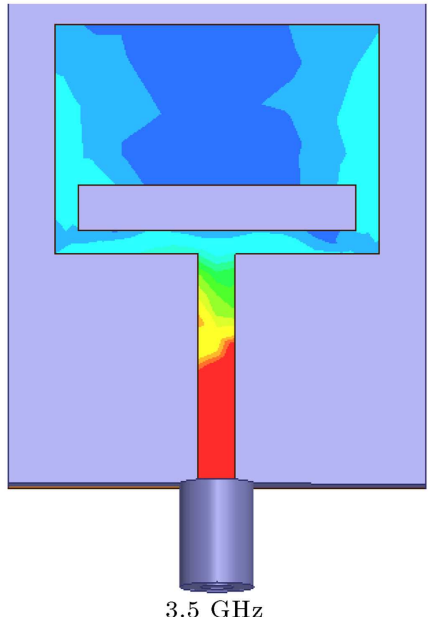

$3.5 \mathrm{GHz}$

Figure 16. Surface current distribution on the radiator in the OFF state. 
directional pattern, sufficiently low cross polarization, and low SAR, the proposed antenna is a suitable candidate for communications systems.

\section{References}

1. Lin, S.C., Jong, T.L., Hsieh, C.W., and Ho, K.J. "Wideband series-fed dipole antenna with balun integrated", J. Electromag. Waves Applicat., 24(17-18), pp. 2463-2477 (2010).

2. Irina, B.V., Alexander, R., Komsan, K., Jiasheng, H., and Dmitry, F. "Ultra-Wideband (UWB) planar antenna with single, dual, and triple-band notched characteristic based on electric ring resonator", IEEE Antennas Propag. Lett., 16, pp. 1597-1600 (2017). DOI: 10.1109/LAWP.2017.2652978

3. Lin, P., Bao-Jian, W., Xiao-Feng, L., Xing, J., and SiMin, L. "CPW fed UWB antenna by EBGs with wide rectangular notched-band", IEEE Access, 4, pp. 95459552 (2016). DOI: 10.1109/ACCESS.2016.2646338

4. Elmobarak, H.A., Rahim, S.K.A., Himdi, M., Castel, X., and Abedian, M. "A transparent and flexible polymer-fabric tissue UWB antenna for future wireless networks", IEEE Antennas Propag. Lett., 16, pp. 1333-1336 (2016). DOI: 10.1109/LAWP.2016.2633790

5. Chandu, D.S. and Karthikeyan, S.S. "A novel broadband dual circularly polarized microstrip-fed monopole antenna", IEEE Trans. Antennas Propag., 65(3), pp. 1410-1415 (2017). DOI: 10.1109/TAP.2016.2647705

6. Li, Q.L., Cheung, S.W., Di, W., and Yuk, T.I. "Optically transparent dualband MIMO antenna using micro-metal mesh conductive film for WLAN systemr", IEEE Antennas Propag. Lett., 16, pp. 920-923 (2016). DOI: 10.1109/LAWP.2016.2614577

7. Latheef, A.S., Chinmoy, S., Jawad, Y.S., and Yahia, M.M.A. "Ultra-wideband monopole antenna for multiband and wideband frequency notch and narrowband applications", IET Microwaves, Antennas Propag., 10(11), pp. 1204-1211 (2016).

8. Jayendra, K. "Compact MIMO antenna", Microwave Optical Technology Lett., 58(6), pp. 1294-1298 (2016).

9. Sudeep, B. and Dinesh, K.V. "Bandwidth enhancement of a planar monopole microstrip patch antenna", Int. J. Micro. Wireless Tech., 1, pp. 1-6 (2014).

10. Hall, P.S., Gardner, P., and Faraone, A. "Antenna requirements for software defined and cognitive radios", Proceedings of the IEEE, 100(7), pp. 2262-2270 (2012).

11. www.ieee802.org/22

12. http://eecs.ceas.uc.edu/ cordeicm/papers/ dyspan05 802-22.pdf

13. Rifaqat, H. and Mohammad, S.S. "Integrated reconfigurable multiple-input-multiple-output antenna system with an ultra-wideband sensing antenna for cognitive radio platforms", IET Microwaves, Antennas Propag., 9(9), pp. 940-947 (2015).
14. Tawk, Y., Bkassiny, M., El-Howayek, G., et al. "Reconfigurable front-end antennas for cognitive radio applications", IET Microwaves, Antennas Propag., 5(8), pp. 985-992 (2010).

15. Tawk, Y., Costantine, J., and Christodoulou, C.G. "Cognitive-radio and antenna functionalities: a tutorial”, IEEE Antennas Propag. Mag., 56(1), pp. 231243 (2014).

16. Gunjan, S., Akhilesh, M., and Ajay, C. "Compact reconfigurable UWB slot antenna for cognitive radio applications", IEEE Antennas Propag. Lett., 16, pp. 1139-1142 (2016). DOI: 10.1109/LAWP.2016.2624736

17. Mun, B., Jung, C., Park, M., and Lee, B. "A compact frequency-reconfigurable multiband LTE MIMO antenna for laptop applications", IEEE Antennas Propag. Lett., 13, pp. 1389-1392 (2014).

18. Lee, S.W. and Sung, Y. "Compact frequency reconfigurable antenna for LTE/WWAN mobile handset applications", IEEE Trans. Antennas Propag., 63(10), pp. $4572-4577$ (2015).

19. Pazin, L. and Leviatan, Y. "Reconfigurable rotated-T slot antenna for cognitive radio systems", IEEE Trans. Antennas Propag., 62(5), pp. 2382-2387 (2014).

20. Ban, Y., Sun, S., Li, P., et al. "Compact eight-Band frequency reconfigurable antenna for LTE/WWAN tablet computer applications", IEEE Trans. Antennas Propag., 62(1), pp. 471-475 (2014).

21. Sung, Y. "Compact quad-band reconfigurable antenna for mobile phone applications", Electronics Lett., 48(16), pp. 977-979 (2012).

22. Erfani, E., Nourinia, J., Ghobadi, C., Niroo-Jazi, M., and Denidni, T.A. "Design and implementation of an integrated UWB/reconfigurable-slot antenna for cognitive radio applications", IEEE Antennas Propag. Lett., 11, pp. 77-80 (2012).

23. Srivastava G., Mohan A., and Chakrabarty A. "Compact reconfigurable UWB slot antenna for cognitive radio applications", IEEE Antennas Propag. Lett., 16, pp. 1139-1142 (2017).

24. Jayendra, K., Talukdar, F.A., and Banani, B. "Frequency reconfigurable E-shaped patch antenna for medical applications", Microwave Optical Technology Lett., 58(9), pp. 2214-2217 (2016).

25. Liping, H., Caixia, W., Xinwei, C., and Wenmei, Z. "Compact frequency reconfigurable slot antenna for wireless application", Antennas Wireless Propag. Lett., 15, pp. 1795-1798 (2016).

26. Yang X., Lin J., Chen G., and Kong F. "Frequency reconfigurable antenna for wireless communications using GaAs FET switch", IEEE Antennas Wireless Propag. Lett., 14, pp. 807-810 (2015).

27. Rajagopalan, H., Kovitz, J.M., and Rahmat-Samii, Y. "MEMS reconfigurable optimized E-shaped patch antenna design for cognitive radio", IEEE Trans. Antennas Propag., 62(3), pp. 1056-1064 (2014).

28. https://www.ieee.li/pdf/essay/pin_diode handbook.pdf 
29. http://www.cadfamily.com/download-pdf/ANSYS_ HFSS_ANSYS_HFSS_L06_ 2_HFSS_3D_bc.pdf

30. Abbasiniazare, S., Manoochehri, O., and Forooraghi, K. "A reconfigurable printed dipole antenna using $\mathrm{RF}$ PIN diodes", Micro. Optical Technology Lett., 56(5), pp. 1151-1155 (2014).

31. Ting, L., Xianjun, H., Kuo, H.C., JiaCing, C., Mahmoud, A.A., and Zhirun, H. "Graphene nanoflakes printed flexible meandered-line dipole antenna on paper substrate for low-cost RFID and sensing applications", IEEE Antennas Propag. Lett., 15, pp. 15651568 (2016).

\section{Biographies}

Jayendra Kumar is currently working as an Associate Professor in the School of Electronics Engineering, VIT Amaravati University, India. He received BE and ME from Solapur University, Maharashtra, India in 2011 and 2014, respectively, and completed PhD from National Institute of Technology Silchar, Assam, India in 2018. He is currently working on the frequency reconfigurable, Reduced Surface Waves (RSWs), and high gain single element antennas. Prior to this, he was working on Electronic Bandgap Structures (EBGs), ultra-wideband antennas, and mutual coupling reduction in Multiple Input Multiple Output (MIMO) antennas. His research interests include dielectric resonator antennas, metamaterials/metasurface, conformal antennas, multi-layered substrates, and higher order modes antennas. He has delivered guest lectures and held Short-Term Training Programs (STTPs) on Microstrip antennas, High-Frequency Structural Simulator (HFSS), Matlab, and solar energy awareness programs. He is a student member of IEEE Antennas and Wave Propagation Society (APS), a member of Indian Society of Technical Education (ISTE), and an active reviewer of the International Journal of Systems, Control, and Communications (IJSCC).
Banani Basu received BE from Jalpaiguri Government Engineering College, India in 2004 and MTech from West Bengal University of Technology, India in 2008, both in Electronics and Communication Engineering. She received her $\mathrm{PhD}$ from National Institute of Technology, Durgapur, India in 2012. Presently, she is an Assistant Professor at the Department of ECE at National Institute of Technology, Silchar, India. She has published 12 research papers in the international journal of repute and 10 papers in international conferences. She is a reviewer of several international journals and a program committee member of many international conferences. Her research interests include array antenna synthesis, soft computing, and electromagnetics.

Fazal Ahmed Talukdar received his BE (Hons) from Regional Engineering College, Silchar (now, NIT Silchar) in 1987. He obtained his MTech in 1993 and $\mathrm{PhD}$ in 2002-2003 from the Indian Institute of Technology Delhi and Jadavpur University, respectively. He joined Regional Engineering College, Silchar as Lecturer in April, 1991, became an Assistant Professor in March 1996, and appointed as a Professor in May, 2006. Prior to joining Regional Engineering College, Silchar, he was a Lecturer at Silchar Polytechnic from August 1988 to March 1991. He was the Head of the Department of Electronics and Communication Engineering from August 2006 to April 2006. During July 2004 to June 2009, he was the Registrar of NIT Silchar. During March 2005 to August 2007, he held the post of Deputy Registrar (Accounts). During 201213, he was the Dean (Alumni Relations); during 201315, he was the Dean (Academic Affairs) of the National Institute of Technology Silchar, India. Besides, he worked in many other Committees of the National Institute of Technology Silchar, India. His research interest includes signal processing, analog circuits, etc. 